\title{
CONTROLLED MULTIMEDIA CLOUD ARCHITECTURE AND ADVANTAGES
}

\author{
SelvaraJ KeSAVAn1, Jerome AnAND2, DR.J.JAYAKUMAR3 \\ 1RESEARCH Scholar, KARUNYA UnIVERsity, CoImbatore, TAMIL NADU, INDIA. \\ SELVARAJKESAVAN@GMAIL . COM \\ 2EMbedDED SOFTWARE ARCHITECT, BANGALORE, KARNATAKA, INDIA. \\ LJEROME@GMAIL . COM \\ 3Assistant Professor, Dept of EEE, Karunya University, CoImbatore, \\ TAMILNADU, INDIA. \\ EMAILTOJAYAKUMAR@GMAIL . COM
}

\begin{abstract}
Cloud computing is the next generation computing platform that offers many applications to the users in seamless way and access the resources across the network without any boundary. Multimedia content management and processing is among the important aspect of the cloud and it brings the advantages of processing and delivering of Multimedia in the distributed environments. Cloud users are having interest to access and share the media within the community by using the devices with limited capability. With current cloud architecture, restrictive multimedia content access and processing within the cloud community is not possible. To overcome this problem, we propose the private controlled cloud architecture for the media which stores, processing and delivering the media content to the authenticated clouders on the go. Also we captured some of the key advantages of this architecture over the existing methods.
\end{abstract}

\section{KEYWORDS}

Streaming, Media cloud, mobility, Controlled cloud, clouders

\section{INTRODUCTION}

With the advent of cloud computing, users will have the flexibility to enjoy media content anywhere in the world and at anytime. The consumer electronic devices influenced by the cloud technology and deliver the state-of-art services to the users. The user just needs to purchase the media content, store it in the cloud and the cloud does the rest. The cloud offers scalable, dynamic resource allocation to the computing environments and abstracts the underlying complexity.

Multimedia applications occupy the key space in the cloud and require consistent support from cloud. Processing multimedia content such as Video, Audio, Image requires complex hardware/software and intensive power requirements. Media applications such as streaming

DOI : 10.5121/acij.2012.3204 
from the cloud will act as an alternative for progressive downloads where the user needs to maintain/store the media content. With the cloud maintaining the purchased data, the users will have a burst of applications to play with. One such application is the streaming of media from the cloud. The cloud takes care of processing/ streaming the media content and the end users dummy client just renders it without the need of any high-end hardware. With all the complexities hidden in the private cloud, the users of the cloud namely the clouders, just have to play with the services that the cloud offers them without any hassles.

In the past, it was difficult for users to get on-demand streaming media services with jitter, reliability, hardware/software processing capabilities and so on. This can be overcome by using various cloud computing methodologies and services. The server in the cloud will take care of the media content processing ranging from mixing, trans-coding and unifying streams from various clouds and so on. The scope of this paper is to managing, controlling and sharing the media across the community cloud and hence we call our cloud as the "media cloud" and the way the users get access to the services from the private cloud is called as "clouding".

In the current trend, multiple companies have started providing content management systems over the cloud like Amazon EC2[7], Amazon Simple Storage Service, Google Music[8], Apple Cloud service, streaming media cloud etc[1][5]. Cloud users can stream the media content and listen the songs anytime with their computers or smart devices. There are also many new applications coming up over the cloud and one of the interesting applications is the "cloud drive" introduced by Amazon for music content usage [6].

The objective of this paper is to provide the platform for control and access the media services with maximum utilization of resources and less user effort without much knowing the underlying technology and complexity. The remaining of the paper is organized as follows Section II briefs the related work carried out in this area. In Section III, we describe the controlled private cloud system architecture, different media cloud services use cases and the key advantages. Finally Section IV concludes the paper with scope of future work.

\section{RELATED WORK}

Cloud computing is the emerging computing platform , many research activities are performed on different sections including platform security[14][15], cloud models[1][5][11], load Balancing[18][19], resource management[20][21], and extend cloud to home[12].The various cloud services ,use cases and key measurement metrics are defined in [1][2][5][10]. Private cloud is a section of the large Public cloud restricted to only a group of users. These users may range from big organizations to small family circle or communities. This model enables the companies have more control over the data and environments.

With these private clouds in place, users will have controlled access to data. This we can call it as the Controlled cloud. What this potentially means is that a super clouder is having an unrestricted access to the private cloud can set restrictions for other users in the cloud similar to IT administrators setting permissions for personal computers.

Media cloud is subsection of cloud paradigm, addresses the cloud infrastructure for various multimedia services, processing requirement and QoS measurement. The article [13] covers the cloud framework requirements and efficient computing models for multimedia applications. Authors in [12] proposed the extension of the media sharing and control from home to cloud computing. Cloud based multimedia services delivery on real time draws attention from many researchers. Authors in [22] captured the challenges in delivering multimedia services over IP network. Compare to the existing cloud framework model, our model proposes the following 
features: 1) the system model for controlled private cloud. 2) Analyse key media cloud services suitable to this model and advantages.

\section{CONTROLLED PRIVATE CLOUD}

Controlled cloud based service provides the clouders with the data and processes being managed within the Group or family without the restrictions of network bandwidth, security and legal requirements that a public cloud service might encounter. It can impose tight control mechanism over all or selected clouders. In addition, private cloud services offer the provider and the user greater control of the cloud infrastructure, improving security and resiliency because user access and the networks used are restricted and designated. Henceforth the private cloud acts like a trusted cloud for a specific clouder community. It follows then, that security and QoS are important concerns to the successful delivery of the service.

In the media space, the private cloud will be the single point of contact for the various clouds where the clouder has bought his cloud service. For instance, if a clouder buys music content from Amazon cloud service provider and a gaming application from Microsoft Corporation, he can have access to both these clouds from a single private cloud which has the authenticity to establish the connection with the other clouds.

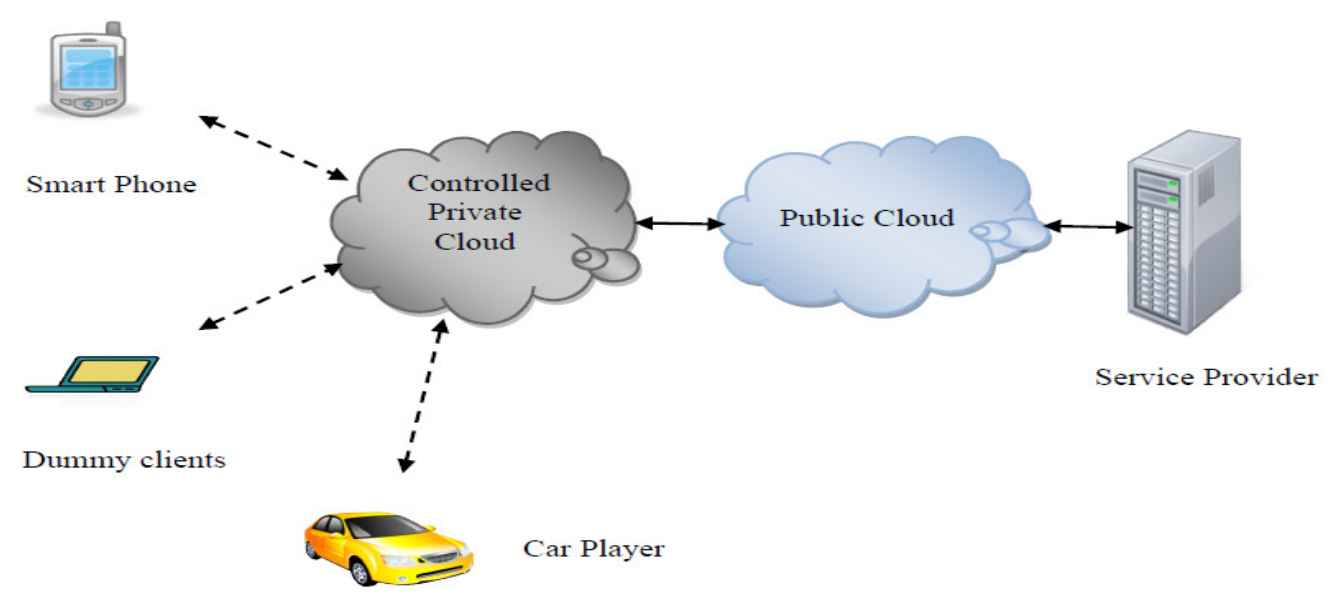

Figure 1 Private controlled cloud

With this private cloud in shape, the clouder can eliminate the use of thumb drives, cables for media content access in a mobile player. Clouds also eliminate the need to transfer media content across devices with the availability of single point of access.

\subsection{The Private Cloud Architecture overview}

The bird's view of the private cloud can be shown as a segregation of three different layers with the application layer interacting with the end-user (clouder). The Segregation comprises of

1. Application Layer

2. Native Processing Layer - the media core 
3. Platform layer

\subsubsection{Application Layer}

The application layer is like an Application Factory containing the different applications bought by the clouder. It manages and maintains the services provided by the installed application. It uses underlying native layer for computing and data storage. The Clouder accesses the service through a set of well defined standard API's.

Some of the core media services such as media streaming, video sharing and access media content are hosted and managed in the Private Controlled Cloud.

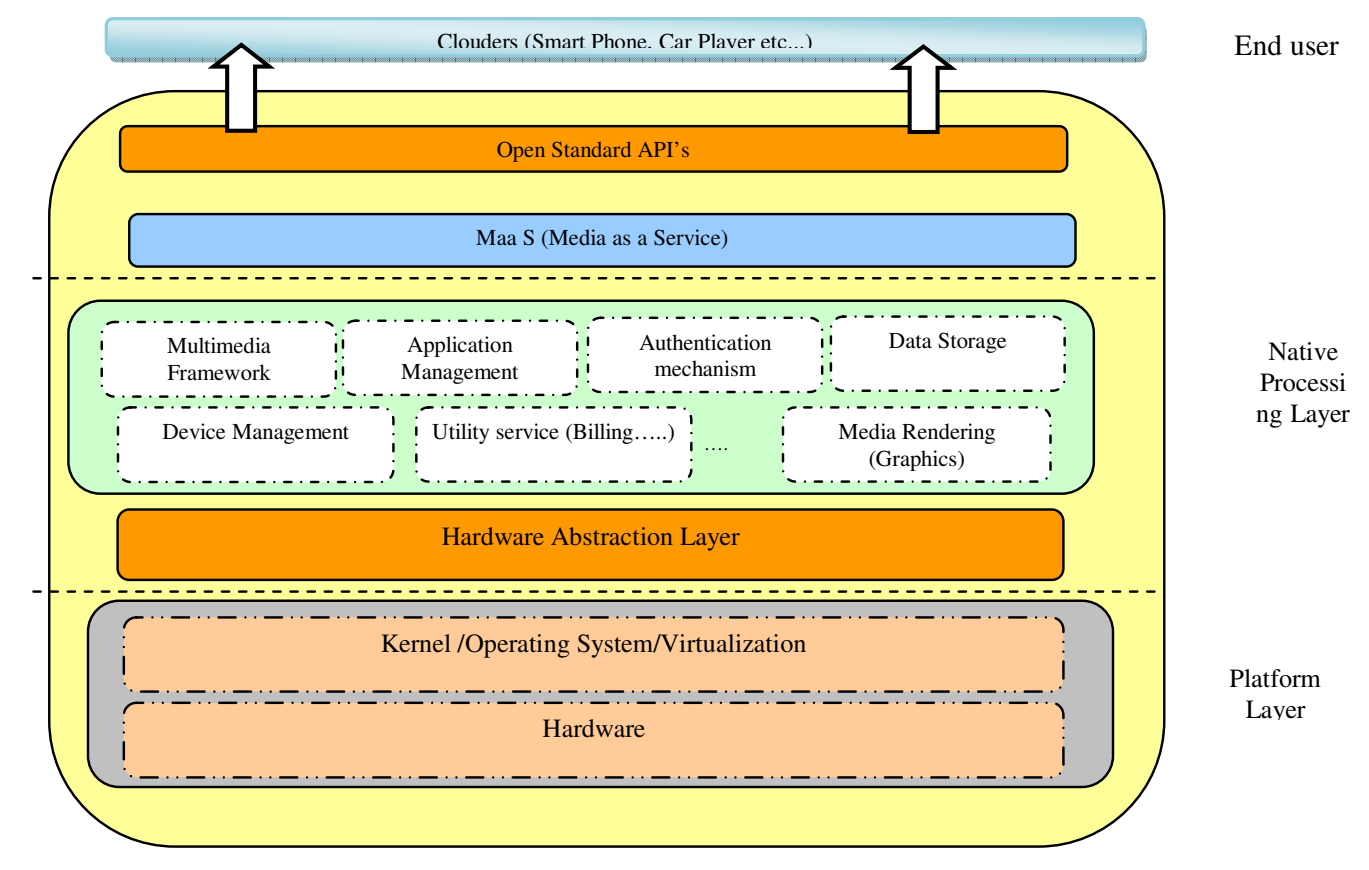

Figure 2 Private Cloud Layered

\subsubsection{Native Processing Layer}

Native processing layer is the core of the private cloud and it provides come of the basic functionalities including

- Data storage

- indexing and storing the application resources and meta data.

- Authentication mechanism - Provides the basic and selected privileges to the Clouder

- Multimedia Framework - Middleware signal and media component to process various multimedia functionalities such as streaming, Audio, Video mixing, media content share etc..

- Application management - Monitor the status of the application component and its resources etc..

- Media Rendering

- rendering of High resolution media content using the complex high performance hardware's of the cloud.

- Utility service

- Metering the service users and extent in which it is used. 
Advanced Computing: An International Journal ( ACIJ ), Vol.3, No.2, March 2012

- Device management - management of multiple clouder's with unique ID's and services based on the request and permission .

The Hardware Abstraction Layer has the platform dependent calls abstracted and mapped with native processing layer functionalities. The function of the native processing layer is not only limited to the media services as mentioned above. The layer can be further extended to other application abstraction logic and we leave it to the reader's imagination.

\subsubsection{Platform Layer}

The Platform layer is one of the critical aspects of the cloud architecture as it contains the complex media processing hardware's which the clouder doesn't have access to. It contains the Kernel, network capability, virtualization mechanism etc based in the hardware. It provides the infrastructure for the cloud to facilitate the different media service such as middleware computing, data processing, storage, decoding, rendering etc. for the media service that the cloud offers.

The responsibilities of the platform layer includes but not limited to

- Hardware resources

- Scheduling/processing mechanism

- Virtualization mechanism

- Hardware media pre/post processing

- Synchronization, etc

\subsection{Media clouds}

The clouds maintaining the media content and which aids in presenting the content to the owner of the cloud using media signaling protocols is the media cloud. For example the Media content from the cloud can be streamed to dummy clients residing in car players/ smart phones etc. This involves streaming of media content, synchronizing them, rendering the content and so on.

Almost all the processing is done over the cloud. With the clouds, it might be possible for the users to share playlists, ratings, etc to the multiple clouders in the private cloud community. Just like the social networking, the users of the cloud can interact with one another, share content without redundant purchases and do lots more. The media cloud with the aid of available streaming protocols like TCP, UDP, RTP, etc will stream the media content to the dummy clients. Restrictions can be imposed on streaming the specified authenticated contents using more proprietary protocols. This will enable the private cloud to stream contents in a controlled environment without the risk of data compromise.

Streaming of media content from the cloud will involve buffering of data, decoding, rendering, mixing and rating them over the standard set of APIs that the application provides. The cloud takes care of the packetizing, profiling and so on based on the streaming protocols. With the streaming application in scope, the private cloud provides the following in line with the basic cloud computing architecture.

SaaS - Streaming media server; this we call it as MaaS (media as a service).

IaaS - The infrastructure needed to store the media content

PaaS - The hardware's needed to decode the content and process it 


\subsection{Media cloud services}

Multimedia refers to information presented in more than one format, such as text, audio, speech, video, graphics, and images. In each of these formats there are a myriad of sub-formats .With rapid changes and advances in the information technology, the increased usage of multimedia services in mobile and automotive sectors is inevitable.

Various multimedia services such as multi-format Audio/Video playback, recorder, Image Editor, streaming, VOIP, Mobile TV, Video telephony and DLNA are part of day to day life. Economic globalization also fosters the spread of varied multimedia applications like

\subsubsection{In-Vehicle Infotainment:}

With Private clouds in place, in-vehicle infotainment over the cloud without client side processing / storage becomes a possibility. Media content is streamed from the cloud to the car media system which will act as a dummy client with basic media rendering capability. A dummy Hands Free module (HFM) can be used in establishing voice based communication with the cloud making the drivers life simpler.

\subsubsection{Telematics}

Telecommunication is possible using Hands Free Modules in the car systems to connect with other users in the cloud without the need of a third party service provider. Push to talk over cellular (PoC) and other fleet management systems also become a possibility with the advent of private clouds. With each user of a private cloud registered to the cloud, both one to one and multiple communications become possible through the cloud. This provides controlled access of data amongst the cloud users.

Moreover, other use cases like multi-line, conferencing, dynamic call applications using any of the different codec's becomes a possibility within the cloud infrastructure.

\subsubsection{IMS applications}

Applications based on IP-Multimedia subsystem can be easily integrated into the cloud architecture. Each clouder will be identified with a unique ID within the cloud subnet. (By the clouder, we mean the end-user with a device access.) This becomes the crux for IMS applications ranging from Voice/ Video over IP, Video Share, to the simple Push to talk applications for fleet vehicles. This IMS architecture in conjunction with the telematics core will make the cloud more users friendly in the near future.

The different multimedia applications can be mix and matched to deliver content to the users in a more refined manner. For instance, the picture content can be edited before streaming to the client using image editor software integrated to the cloud. VOIP, PTT services can be made to include voice editing software at the clouds end without having the need of that complex software's at the client side. Some of the use cases and applications which can be integrated to the private cloud are shown in the diagram below 


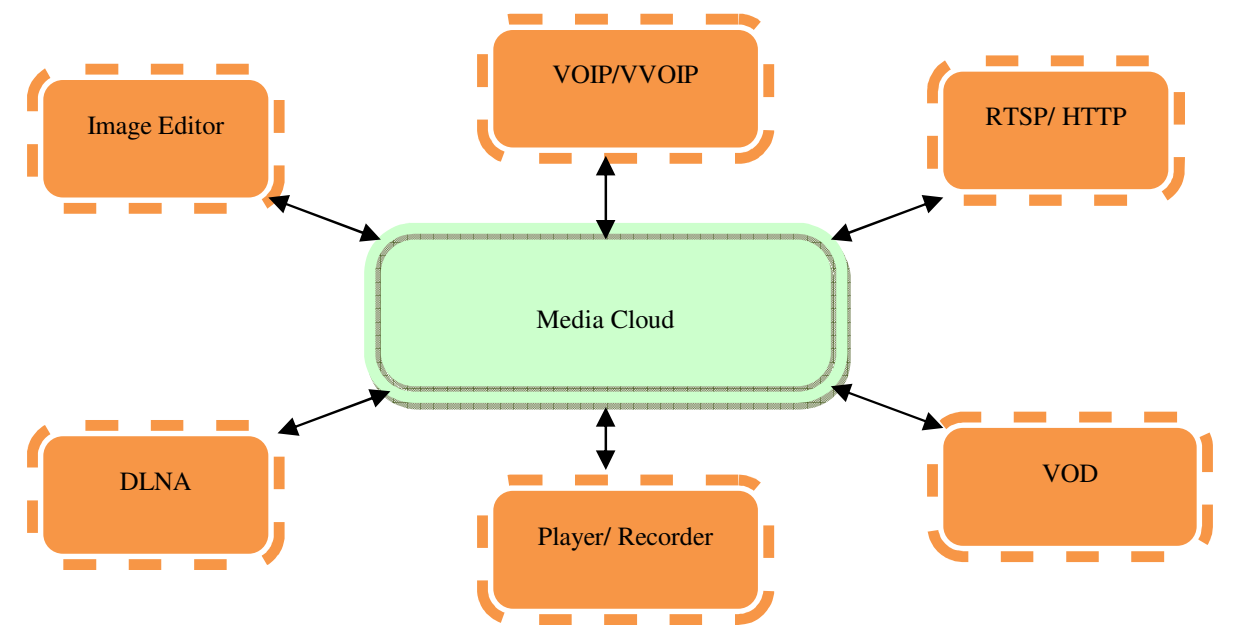

Figure 3 Media cloud Services

\subsubsection{Cloud gaming}

Complex and high end games can be integrated to the cloud and given access to the users. This will enhance the existing gaming networks currently available. All the complex high-end processing can be done over the cloud and the user provided with just the controls for the games using client software and displaying the processed data to the client's dummy terminals.

\subsection{Media clouding}

The media cloud can further be enhanced to network with multiple users and clouds much like the social networking concept. This allows access of data content across multiple users and across multiple clouds. This sharing of media content and access of the data by the user based on web based queries is called as the media clouding.

The media clouds can form a network of clouds using gateways much like the home gateway of different devices. This will involve registration, authentication and secured communication among the clouds.

\subsubsection{Cloud gateways}

Cloud gateways are high end hardware's which provide the authentication and registration mechanism for the private cloud. They can be considered as modern day firewalls for the cloud. The play a pivotal part in the clouding mechanism thereby aiding the private cloud owners share data between the clouds in the controlled, safe environment. This conglomeration of the private cloud and the gateway is described as the controlled cloud in the above sections. 


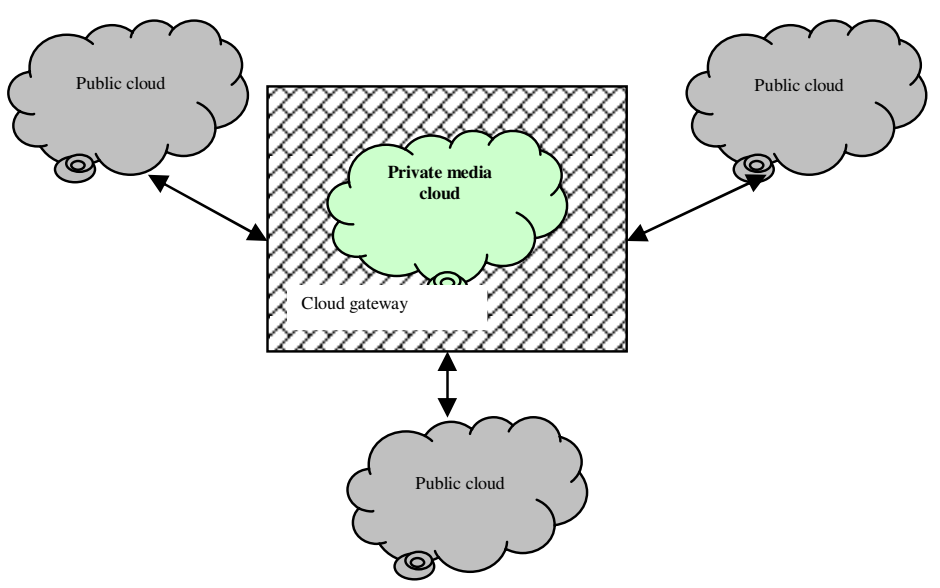

Figure 4 Cloud gateway

\subsubsection{Load-balancing over the cloud}

With the sharing of content between clouds, it would also be possible to share the workload amongst the clouds. This will reduce the processing time in a particular cloud. Similar to the load balancing concepts in the network routing technologies, we can have the cloud balance the work load based on work weights and streaming requirements.

Now when it comes to sharing the media content to the user in conjunction with load sharing, there can be many hazards posed to the media content to be streamed. Some of the key challenges would involve delay in sharing data, synchronization of media data processed across clouds and so on. More research and development needs to be done in this are to make the data more readily available for the users.

\subsection{Advantages of using the cloud}

The private cloud offers a series of advantages for the different multimedia applications and some of the critical ones are explained through the below sections

\subsubsection{Standard Application Programming Interface}

Using Private cloud architecture, we can provide the standard application programming interfaces for the clouders and developers in need of the media contents in the cloud. This will help to create a unique, standard programming model and avoid duplication of API's for the users who want to work on cloud computing architecture.

\subsubsection{Data Storage}

Private cloud elements interact with the public cloud for registering and receiving the services. In case of a media service, once the Private cloud receives the media content/stream, it stores the content in the private cloud. The private cloud also provides controlled delivery of the data in a fast and efficient way from its local storage. Data indexing in addition to different clouding logics is one of the key elements involved in providing fast access of media content for the clouders.

Data Maintenance is also carried out at specific time intervals over the private cloud. This is related to the server down time and it will lead to multiple complexities which are not covered in this paper. 
Advanced Computing: An International Journal ( ACIJ ), Vol.3, No.2, March 2012

\subsubsection{Strong Authentication Mechanism}

Due to increasing user demands, there is an inevitable requirement to provide selective permissions and authentication to clouders. It is not possible to have the controlled permission for the clouders in the public Cloud. Private clouds enable the authentication to the clouders in such a way that they can share and stream audio/video/image amongst themselves.Also it can impose restricted permissions to selected clouders.

For example the children can view only selected media content and fully privileged access can be given to other users.

\subsubsection{Cost Effective solution}

With the clouders not needing to maintain or purchase new hardware, the cost gets reduced as a whole, as the single unified hardware/ software are shared across the clouders.

\subsubsection{Network Bandwidth usage}

Network bandwidth is one of the most critical factors for delivering media content with acceptable QoS. The QoS for a specific media service is measured in terms of transit time delay, error rate, synchronization, caching efficiency, response time etc. The Private cloud aids in reducing the network bandwidth as it is isolated from the public cloud space. There can be algorithms to minimize the resource usage between private and public cloud by unifying all the private cloud user requests into one. The private cloud uses its own data space for storing the media content, clouder's gets service without any restriction of the bandwidth. The data also will be less prone to the channel error with the usage of private clouds as a single point of contact for multiple clouders of the same user space (community/ organization).

\subsubsection{Dynamic Resource Allocation}

The private cloud architecture enables the on-demand run time resource utilization based on the type of service. For media services, the signaling and media components are used dynamically to build the engine for various applications such as audio/video editing, video telephony, VOIP etc.

\subsubsection{Controllability}

This paper provides insight on how the cars can be connected to the cloud. This would provide a personalized touch to the cars which we use in our daily lives. With technologies and software coming up, the cars might be controlled from an individual's home. This will pave way for more controlled applications in the field of electronics.

\subsubsection{Common platform for multimedia applications}

The private cloud architecture can pave way for a common base for the various multimedia applications of the future where the processing is done at the cloud end. This can also provide single stop facility for different networking architectures like circuit switched, mobile networks and so on. This can be extended to IMS applications which will have the single base application service platform for the various networks to converge to. This can be shown diagrammatically in figure 5 .

With this common platform in place, communication, data, quality etc can be extended from the infrastructure as a service (IaaS) entity of the cloud topology. Hence Communication as a service (CaaS), data as a service (DaaS) etc can grow from the usage of common cloud platforms. 


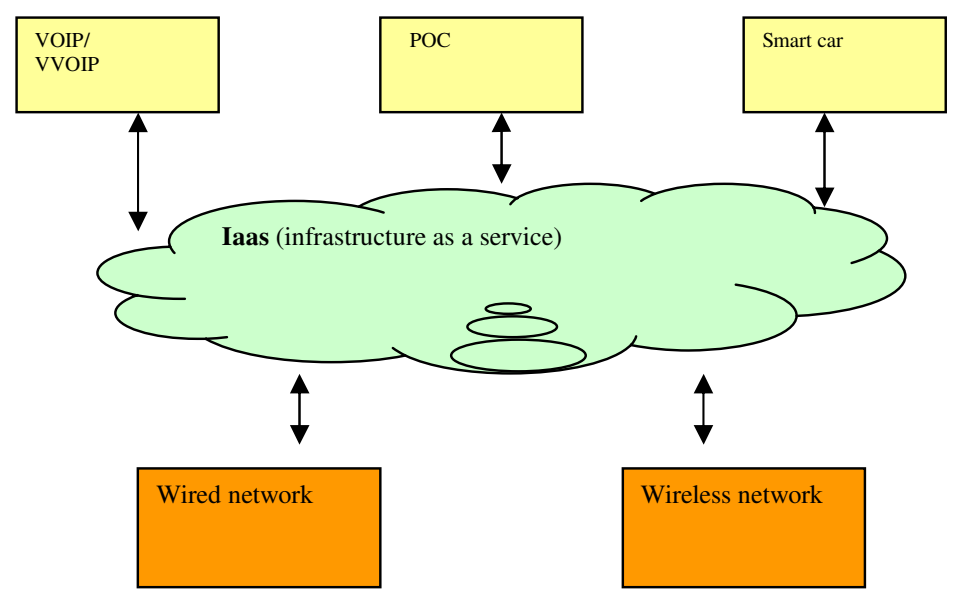

Figure 5 Common platform support

\subsection{Media clouding: Key Challenges}

Some of the main challenge for the mobility of media content using the cloud involves data reliability, network availability, load imbalance and data latency/ synchronization in case of multiple media streams from different clouds. Also, if the media cloud processes the media content before streaming, challenges in streaming the processed content to the dummy clients using the existing streaming protocols will not be sufficient thereby calling for enhancements in the streaming protocols

\section{CONCLUSION}

In this paper, we have analysed the multimedia content data processing and control problem for the different multimedia cloud applications within the community. We propose the new model, controlled private cloud architecture for sharing media content to the clouders and some of the more exciting clouding multimedia applications possible in the near future. As more and more clouders can join the cloud, media clouding will provide a range of applications in a controlled environment. We have also discussed some of the extensive use cases and advantages of the private cloud paving the way for further research and development. This paper will also aid in further research and enhancement for the current streaming/ networking protocols in place for the multimedia contents.

\section{REFERENCES}

[1] James F. Ransome, John W.Rittinghouse, "Cloud Computing Implementation, Management \&Security, CRC Press, 2010.

[2] Barrie Sisisky,” Cloud Computing Bible”. John Wiley \& Sons, January 11, 2011.

[3] Amreen Khan and KamalKant Ahirwar," Mobile cloud computing as a future of mobile multimedia database". International Journal of Computer Science and Communication, Vol. 2, No. 1, January-June 2011, pp. 219-221.

[4] Weiguang Song and,Xiaolong Su, "Review of Mobile cloud computing “.2011 International conference on Communication software and Networks(ICCSN), May 2011. 
Advanced Computing: An International Journal ( ACIJ ), Vol.3, No.2, March 2012

[5] George Reese ,"Cloud Application Architecture” O’Reilly publication.

[6] Amazon Cloud Drive. [Online].Available: http://en.wikipedia.org/wiki/Amazon_Cloud_Drive

[7] Amazon elastic compute cloud. [Online]. Available:http://aws.amazon.com/ec2

[8] Google app engine. [Online]. Available:http:///code.google.com/appengine

[9] Amazon cloud front.[Online].Available: http://aws.amazon.com/cloudfront

[10] Cloud computing use cases. [Online]. Available: http://www.scribd.com/doc/18172802/Cloudcomputing-Use-Cases-Whitepaper

[11] Introduction to Cloud Computing Architecture, White paper, SUN, Microsystems, 1st edition, June 2009.

[12] Diaz-Sanchez, D., Almenarez, F.,Marin, A,Proserpio, D. Cabarcos, P.A.," Media cloud: an open cloud computing middleware for content management" IEEE Transactions on Consumer Electronics ,May 2011,issue2 970-978

[13] W. Zhu, C. Luo, J. Wang, and S. Li, "Multimedia cloud computing: Directions and applications," Special Issue on Distributed Image Processing and Communications, IEEE Signal Processing Magazine, May 2011.

[14] D. Huang, X. Zhang, M. Kang, and J. Luo, "MobiCloud: Building Secure Cloud Framework for Mobile Computing and Communication," in Proc. IEEE International Symposium on Service Oriented System Engineering, 2010, pp. 27-34.

[15] W. Lu, A. Varna, and M. Wu, "Security analysis for privacy preserving search of multimedia," in Proc. IEEE International Conference on Image Processing (ICIP), 2010, pp. 2093-2096.

[16] G. Li, H. Sun, H. Gao, H. Yu, and Y. Cai, "A survey on Wireless Grids and Clouds," in Proc. IEEE International Conference on Grid and Cooperative Computing, 2009, pp. 261-267

[17] B. Rimal, E. Choi, and I. Lumb, "A taxonomy and survey of cloud computing systems," in Proc. IEEE Fifth International Joint Conference on INC, IMS and IDC, 2009, pp. 44-51.

[18] Hui Wen,Zhao Hai-ying,Lin Chuang,Yang Yang:” Effective load balancing for cloud-based multimedia system" 2011 International Conference on Electronic \& Mechanical Engineering and Information Technology, 165 - 168, 19 September 2011.

[19] S. Wang, K. Yan, et al., "Towards a Load Balancing in a three-level cloud computing network, "Computer Science and Information Technology (ICCSIT), 2010 3rd IEEE International Conference on,vol. 1,2010, pp. 108-113.

[20] W. Lin and D. Qi, "Research on Resource Self-Organizing Model for Cloud Computing," in Proc. IEEE International Conference on Internet Technology and Applications, 2010, pp. 1-5.

[21] F. Teng and F. Magoules, "Resource Pricing and Equilibrium Allocation Policy in Cloud Computing," in Proc. IEEE International Conference on Computer and Information Technology (CIT 2010), 2010, pp. 195-202. .

[22] Kai-Di Chang, Chi-Yuan Chen, Jiann-Liang Chen and Han-Chieh Chao, "Challenges to Next Generation Services in IP Multimedia Subsystem" .Journal of Information Processing Systems, Vol.6, No.2, June 2010.

[23] O. Shoewu, "Service Delivery in Multimedia Traffic Communications". The Pacific Journal of Science and Technology, Volume 10. Number 2. November 2009

[24] W. Lin and D. Qi, "Research on Resource Self-Organizing Model for Cloud Computing," in Proc. IEEE International Conference on Internet Technology and Applications, 2010, pp. 1-5.

[25] F. Teng and F. Magoules, "Resource Pricing and Equilibrium Allocation Policy in Cloud Computing," Proceeding in IEEE International Conference on Computer and Information Technology (CIT 2010), 2010, pp. 195-202. 
Advanced Computing: An International Journal ( ACIJ ), Vol.3, No.2, March 2012

[26] Judith Hurich, Robin Bloor, Marcia Kaufman, Fern Halper,'Cloud Computing for Dummies" Wiley Publication Inc., 2010.

[27] Tom White," Hadoop the Definite Guide”, O’REILLY, 2009.Department of Information Technology 23

[28] Tim Mather, Subra Kumarasamy, Shahed Latif," Cloud Security and Privacy”, O’REILLY, 2009.

[29] Hui Wen,Zhao Hai-ying,Lin Chuang, Yang Yang:” Effective load balancing for cloud-based multimedia system" 2011 International Conference on Electronic\& Mechanical Engineering and Information Technology, 165 - 168, 19 September 2011

[30] Selvaraj kesavan, chandrasekar sakthivel" Unified Approach for Next Generation Multimedia Based Communication Components Integration with Signaling and Media Processing

Framework” International Conference on Signal Acquisition and Processing, November 2010. 